BMJ

Open

Gastroenterology

\title{
Ambulatory care management of 69 patients with acute severe ulcerative colitis in comparison to 695 inpatients: insights from a multicentre UK cohort study
}

\author{
Shaji Sebastian (D) , ${ }^{1,2}$ Kamal V Patel (D) ," Jonathan P Segal (D) , ${ }^{4}$ \\ Sreedhar Subramanian (D) , 5,6 Thomas Edward Conley (1) ,7 \\ Haidee Aleman Gonzalez (1) , ${ }^{1}$ Alexandra J Kent (D) , ${ }^{8}$ Aamir Saifuddin (D) ,9 \\ Lucy Hicks (D) , ${ }^{10}$ Shameer Mehta (D) , ${ }^{11}$ Neeraj Bhala (D) , ${ }^{12,13}$ \\ Matthew J Brookes (D) , ${ }^{14,15}$ Christopher A Lamb (D) ,16,17' \\ Nicholas A Kennedy (D) , ${ }^{18,19}$ PROTECT ASUC collaborators, Gareth J Walker (i) 20
}

\begin{abstract}
To cite: Sebastian S, Patel KV, Segal JP, et al. Ambulatory care management of 69 patients with acute severe ulcerative colitis in comparison to 695 inpatients: insights from a multicentre UK cohort study. BMJ Open Gastro 2022;9:e000763. doi:10.1136/ bmjgast-2021-000763

- Additional supplemental material is published online only. To view, please visit the journal online (http://dx.doi. org/10.1136/bmjgast-2021000763).
\end{abstract}

SS, KVP and JPS are joint first authors.

Received 15 August 2021 Accepted 8 November 2021

Check for updates

(c) Author(s) (or their employer(s)) 2022. Re-use permitted under CC BY-NC. No commercial re-use. See rights and permissions. Published by BMJ.

For numbered affiliations see end of article.

Correspondence to Professor Shaji Sebastian; Shaji.sebastian4@nhs.net

\section{ABSTRACT}

Introduction Acute severe ulcerative colitis (ASUC) traditionally requires inpatient hospital management for intravenous therapies and/or colectomy. Ambulatory ASUC care has not yet been evaluated in large cohorts.

Aims We used data from PROTECT, a UK multicentre observational COVID-19 inflammatory bowel disease study, to report the extent, safety and effectiveness of ASUC ambulatory pathways.

Methods Adults ( $\geq 18$ years old) meeting Truelove and Witts criteria between 1 January 2019-1 June 2019 and 1 March 2020-30 June 2020 were recruited to PROTECT. We used demographic, disease phenotype, treatment outcomes and 3-month follow-up data. Primary outcome was rate of colectomy during the index ASUC episode. Secondary outcomes included corticosteroid response, time to and rate of rescue or primary induction therapy, response to rescue or primary induction therapy, time to colectomy, mortality, duration of inpatient treatment and hospital readmission and colectomy within 3 months of index flare. We compared outcomes in three cohorts: (1) patients treated entirely in inpatient setting; ambulatory patients subdivided into; (2) patients managed as ambulatory from diagnosis and (3) patients hospitalised and subsequently discharged to ambulatory care for continued intravenous steroids.

Results $37 \%$ (22/60) participating hospitals used ambulatory pathways. Of 764 eligible patients, 695 (91\%) patients received entirely inpatient care, $15(2 \%)$ patients were managed as ambulatory from diagnosis and $54(7 \%)$ patients were discharged to ambulatory pathways. Aside from younger age in patients treated as ambulatory from diagnosis, no significant differences in disease or patient phenotype were observed. The rate of colectomy $(15.0 \%$ $(104 / 695)$ vs $13.3 \%(2 / 15)$ vs $13.0 \%(7 / 54)$, respectively, $\mathrm{p}=0.96$ ) and secondary outcomes were similar among all three cohorts. Stool culture and flexible sigmoidoscopy were less frequently performed in ambulatory cohorts.
Summary box

What is already known about this subject?

- Acute severe ulcerative colitis (ASUC) traditionally involves inpatient hospital stay.

What are the new findings?

- This study reports on the largest cohort of ASUC patients managed in ambulatory pathways emerging in UK clinical practice.

- Outcomes from two types of ambulatory pathways are reported on, patients managed as ambulatory from diagnosis and patients hospitalised and subsequently discharged to ambulatory care for continued intravenous steroids.

- Results from this study suggest the potential for considering ambulatory pathways in ASUC management and the need for randomised studies.

How might it impact on clinical practice in the foreseeable future?

- These data may prompt further research into potential development of ambulatory care in ASUC.

- If evaluated to be safe in further prospective studies, ambulatory care may be transformational in the care of ASUC.

Forty per cent of patients treated as ambulatory from diagnosis required subsequent hospital admission. Conclusions In a post hoc analysis of one of the largest ASUC cohorts collected to date, we report an emerging UK ambulatory practice which challenges treatment paradigms. However, our analysis remains underpowered to detect key outcome measures and further studies exploring clinical and cost-effectiveness as well as patient and physician acceptability are needed.

Trial registration number NCT04411784. 


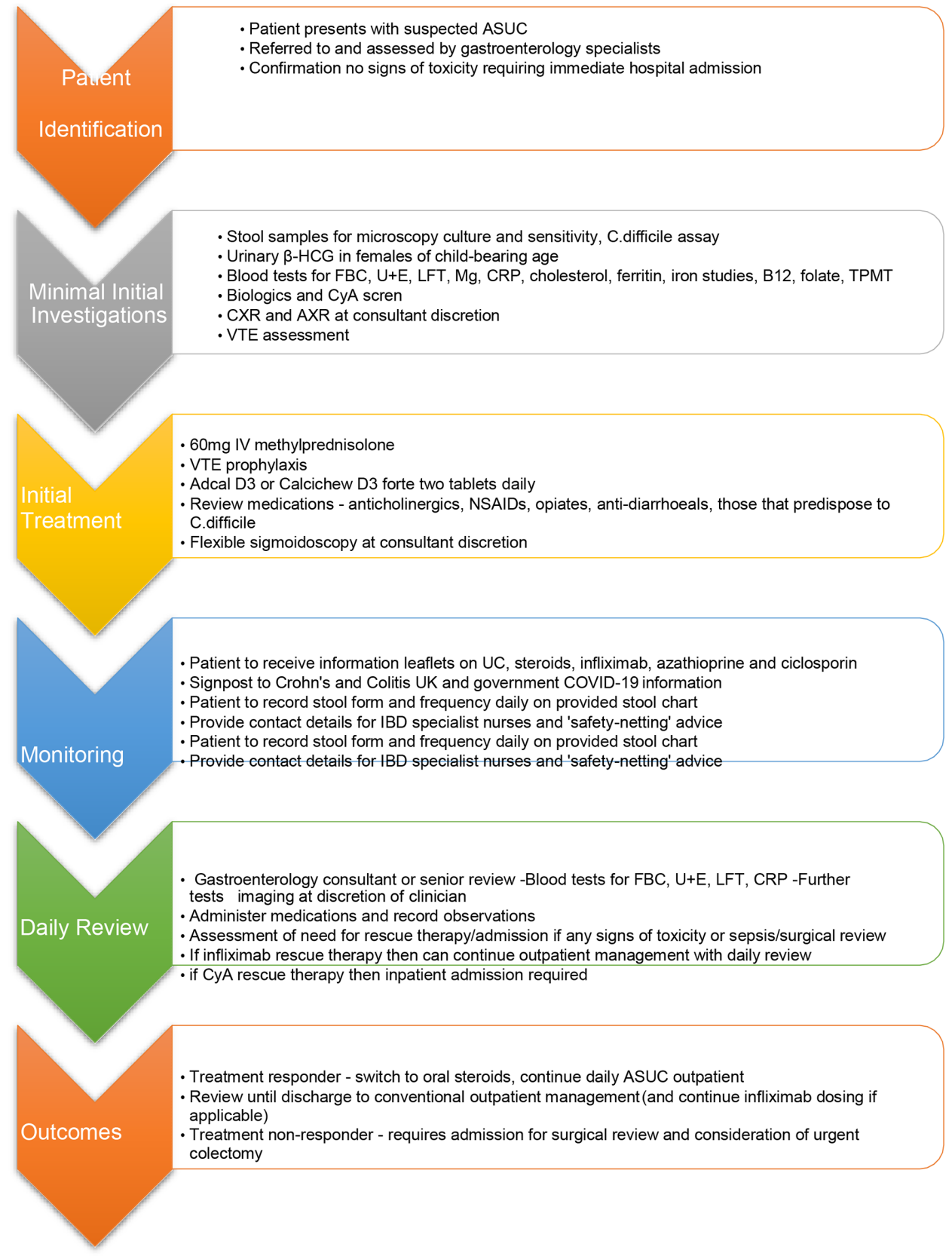

Figure 1 Example of ambulatory ASUC care pathway used during COVID-19 pandemic from Liverpool university hospital Foundation NHS trust and hull university teaching hospitals. ASUC, acute severe ulcerative colitis; CRP, C reactive protein; UC, ulcerative colitis; NHS, National Health Service; HCG, Human Chorionic Gonadotopin; FBC, Full Blood Count; LFT, Liver Function Tests; TPMT, Thopurine Methyl Transferase; CXR, Chest X-Ray; AXE, Abdominal X-Ray; VTE, Venous Thromboembolism; CyA, Cyclosporin A; NSAID, Non-steroidal anti Inflamatory agents.

\section{INTRODUCTION}

Ulcerative colitis (UC) is a chronic relapsing and remitting disease whose aetiology is thought to involve an intricate interplay between host genetics, environment, gut microbiome and immune system. Approximately $15 \%-30 \%$ of UC patients require admission with acute severe UC (ASUC) in their lifetime; for 10\%-15\% this is the first manifestation of their disease. ${ }^{1}$ ASUC is a medical emergency and associated with a mortality of approximately $1 \% .^{2}$ Traditionally, patients are admitted to hospital to facilitate endoscopic assessment, exclude concomitant infective complications, monitor response to first-line corticosteroid treatment and determine the need for and timing of rescue therapy and/or colectomy. Patients with ASUC can deteriorate rapidly and hence require close monitoring of vital signs with correlation to clinical, biochemical and radiological investigations. ${ }^{3}$

The COVID-19 pandemic has placed considerable strain on UK inflammatory bowel disease (IBD) services, necessitating the adoption of measures to facilitate the safe administration of drug infusions, deliver outpatient consultations and avoid nosocomial infections. ${ }^{4}$ Ambulatory care pathways, which use outpatient monitoring and drug delivery, have been shown to deliver safe and 


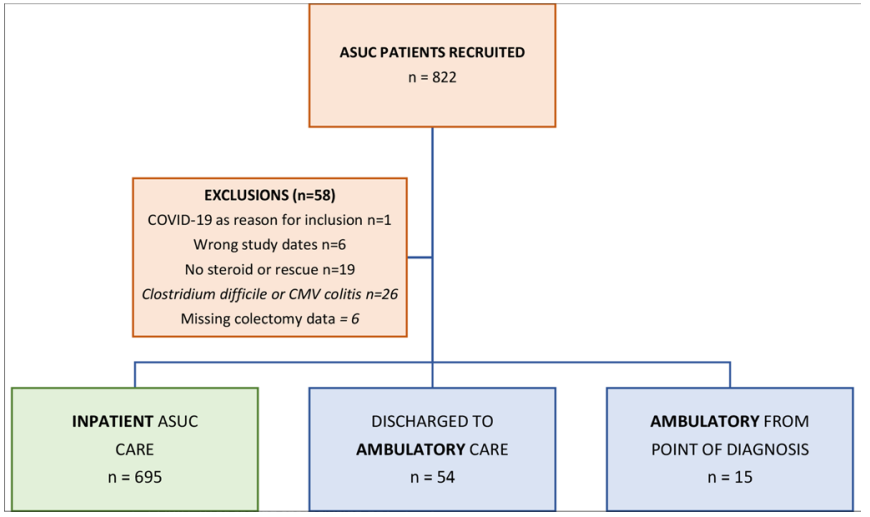

Figure 2 Study cohorts. ASUC, acute severe ulcerative colitis; CMV, cytomegalovirus.

effective treatment for conditions which have historically mandated hospitalisation, for example, pulmonary embolus. ${ }^{56}$ To date, there are a paucity of data regarding the use of ambulatory pathways in ASUC cohorts.

PROTECT-ASUC (Assessment, endoscopy and treatment in patients with ASUC during the COVID-19 pandemic) is a multicentre UK observational study comparing ASUC treatment strategies in patients from a pre-COVID-19 pandemic period with those from the COVID-19 era. ${ }^{7}$ It is one of the largest series of ASUC patients ever collected and has relevance to the contemporary management of UC beyond the COVID-19 pandemic. We previously reported an increase in the use of ambulatory ASUC pathways in COVID-19 era as compared with the historic pre-COVID-19 period, although notably several UK centres had already adopted such practices prior to the pandemic. ${ }^{7}$ We sought to use data from this study to report the extent, safety and effectiveness of ASUC treatment among patients receiving care in ambulatory and traditional inpatient settings.

\section{METHODS}

\section{Study cohorts}

We utilised PROTECT data from 60 acute secondary care UK hospitals which compared ASUC outcomes during the first wave of the COVID-19 pandemic in 2020 with a prepandemic cohort from 2019. Inclusion criteria included all the following: adults aged $\geq 18$ years old; ASUC fulfilling Truelove and Witts criteria; and ASUC diagnosis between either 1 March 2020 and 30 June 2020 (COVID-19 pandemic period) or 1 January 2019 and 30 June 2019 (prepandemic period). Patients with Crohn's disease and cytomegalovirus or Clostridium difficile infections were excluded.

For this post hoc analysis, we combined PROTECT data from the pandemic and prepandemic periods. The decision whether a patient was suitable for inpatient or ambulatory care was left to discretion of the treating physician. We noted ambulatory care consisted of two distinct treatment pathways which we felt warranted separation. Thus, we compared ASUC outcomes in three treatment groups: (1) patients treated entirely in a traditional inpatient hospital setting; and ambulatory patients who received at least one intravenous corticosteroid dose in the outpatient setting subdivided into (2) those patients initially hospitalised and then subsequently discharged to an ambulatory outpatient pathway to continue intravenous corticosteroids and (3) those patients managed as ambulatory from diagnosis of ASUC. An example ambulatory care pathway adapted from the pathways used by Liverpool University Hospital Foundation NHS Trust and Hull University Teaching Hospitals is shown in figure 1; pathways used in other centres likely differed. Centres were encouraged to recruit all patients with ASUC fulfilling the inclusion criteria by interrogating local IBD-database and hospital admission data.

\section{Data collection}

We used baseline clinical information including demographics (age, gender, ethnicity, body mass index, comorbidities and smoking status), disease characteristics (disease duration and disease extent) as well as disease severity markers (C reactive protein (CRP), albumin, haemoglobin, lymphocytes and faecal calprotectin). Following diagnosis of ASUC, details of corticosteroid therapy including preparation, dose, duration, clinical setting where instituted and continued (ambulatory outpatient care or inpatient), need for rescue or primary induction therapy, and emergency colectomy during index admission were used. Follow-up data at 3 months from index ASUC event included subsequent UC flare, subsequent need for hospital readmission for UC flare and subsequent need for colectomy. The day of initial admission was marked as day 1 , or in the case of patients managed entirely in the ambulatory setting, the earlier of first day intravenous corticosteroids or rescue therapy.

All clinical data were collected pseudoanonymised and entered into a secure central REDCap (Research Electonic Data Capture) server hosted at the Royal Devon and Exeter NHS Foundation Trust, UK.

\section{Outcomes of interest}

The primary outcome was the proportion of patients with ASUC requiring colectomy during the index ASUC management period. Secondary outcome measures included need and time to rescue therapy (including primary induction), time to colectomy, total number of days spent in hospital as inpatient (excluding purely ambulatory patients never admitted to hospital), mortality and 3-month follow-up data including: proportion of patients with a further UC flare; proportion of patients readmitted to hospital with UC flare and primary colectomy rates.

\section{Statistical analysis}

We report categorical variables as frequency (\%) and analysed by Fisher's exact test. Continuous variables are summarised as median (IQR) and differences between the three cohorts were analysed using the Kruskal-Wallis test. The study was analysed and reported according to 
Table 1 Baseline characteristics, IBD phenotype and biomarkers at ASUC diagnosis

\begin{tabular}{|c|c|c|c|c|c|}
\hline \multirow[b]{2}{*}{ Variable } & \multirow[b]{2}{*}{$\mathbf{N}$} & \multirow[b]{2}{*}{$\begin{array}{l}\text { Inpatients } \\
\mathrm{n}=695\end{array}$} & \multicolumn{2}{|l|}{$\begin{array}{l}\text { Ambulatory } \\
n=69\end{array}$} & \multirow[b]{2}{*}{$P$ value } \\
\hline & & & $\begin{array}{l}\text { Ambulatory from } \\
\text { diagnosis } \\
n=15\end{array}$ & $\begin{array}{l}\text { Initially inpatient and then } \\
\text { discharged to ambulatory } \\
\mathrm{n}=54\end{array}$ & \\
\hline Sex & 764 & $50.1 \%(348 / 695)$ & $53.3 \%(8 / 15)$ & $38.9 \%(21 / 54)$ & \multirow[t]{2}{*}{0.27} \\
\hline M & 764 & $49.9 \%(347 / 695)$ & $46.7 \%(7 / 15)$ & $61.1 \%(33 / 54)$ & \\
\hline Age (years) & 764 & $37.0(26.0-53.0)$ & $30.0(22.0-39.0)$ & $44.5(33.0-58.8)$ & 0.02 \\
\hline \multicolumn{6}{|l|}{ IBD type } \\
\hline UC & 764 & $95.7 \%(665 / 695)$ & $93.3 \%(14 / 15)$ & $92.6 \%(50 / 54)$ & \multirow[t]{2}{*}{0.34} \\
\hline IBD-U & 764 & $4.3 \%(30 / 695)$ & $6.7 \%(1 / 15)$ & $7.4 \%(4 / 54)$ & \\
\hline BMI & 424 & $24.4(21.2-27.9)$ & $24.0(20.4-28.9)$ & $24.9(22.8-30.9)$ & 0.31 \\
\hline \multicolumn{6}{|l|}{ No of comorbidities } \\
\hline 0 & 764 & $72.2 \%(502 / 695)$ & $60.0 \%(9 / 15)$ & $64.8 \%(35 / 54)$ & \multirow[t]{4}{*}{0.45} \\
\hline 1 & 764 & $19.3 \%(134 / 695)$ & $26.7 \%(4 / 15)$ & $22.2 \%(12 / 54)$ & \\
\hline 2 & 764 & $5.5 \%(38 / 695)$ & $6.7 \%(1 / 15)$ & $7.4 \%(4 / 54)$ & \\
\hline$>2$ & 764 & $3.0 \%(21 / 695)$ & $6.7 \%(1 / 15)$ & $5.6 \%(3 / 54)$ & \\
\hline Years since diagnosis & 722 & $1.0(0.0-5.0)$ & $1.0(0.0-7.5)$ & $1.5(0.0-8.2)$ & 0.85 \\
\hline \multicolumn{6}{|l|}{ UC extent } \\
\hline $\mathrm{E} 1$ - proctitis & 693 & $8.5 \%(54 / 636)$ & $8.3 \%(1 / 12)$ & $4.4 \%(2 / 45)$ & \multirow[t]{3}{*}{0.53} \\
\hline E2-left-sided disease & 693 & $47.3 \%(301 / 636)$ & $41.7 \%(5 / 12)$ & $60.0 \%(27 / 45)$ & \\
\hline E3-pan-colitis & 693 & $44.2 \%(281 / 636)$ & $50.0 \%(6 / 12)$ & $35.6 \%(16 / 45)$ & \\
\hline \multicolumn{6}{|l|}{ Biomarkers at day 1} \\
\hline Stool frequency & 612 & $10.0(8.0-15.0)$ & $12.0(10.0-14.8)$ & $10.0(7.0-13.5)$ & 0.42 \\
\hline CRP & 704 & $54.0(20.0-114.8)$ & $40.0(14.0-72.2)$ & $52.5(20.0-114.5)$ & 0.32 \\
\hline Albumin & 664 & $35.0(30.0-40.0)$ & $38.0(35.0-41.2)$ & $37.0(30.5-39.5)$ & 0.39 \\
\hline Haemoglobin & 704 & $123.0(107.0-135.5)$ & $134.0(113.5-144.5)$ & $129.0(110.0-141.0)$ & 0.15 \\
\hline $\begin{array}{l}\text { Most recent faecal calprotectin } \\
\text { prior to flare }(\mathrm{ug} / \mathrm{g})\end{array}$ & 229 & $501.5(155.8-1364.0)$ & $390.0(78.0-416.0)$ & $600.0(244.8-1234.2)$ & 0.62 \\
\hline
\end{tabular}

$P$ value=Fisher's exact test or Kruskal-Wallis test for discrete and continuous variables continuous, respectively. Discrete variables displayed \% ( $\mathrm{n} / \mathrm{N})$ and continuous variables median (IQR).

ASUC, acute severe colitis; BMI, body mass index; CRP, C reactive protein; IBD, inflammatory bowel disease; UC, ulcerative colitis.

Strengthening the Reporting of Observational Studies in Epidemiology methodology and Statistical Analysis and Methods in the Published Literature. Kaplan-Meier survival curves were plotted for (1) colectomy rates in the first 30 days after diagnosis of ASUC and (2) rescue therapy or colectomy. The combined outcome of rescue therapy (including primary induction) or colectomy was necessary in preference to using rescue therapy alone to avoid the incorrect assignment of patients who went straight-to-surgery as having survived without rescue therapy, when no such therapy would be possible. All tests were two-sided and $p$ values of less than 0.05 were considered to indicate a significant difference, with no correction made for multiple tests. No a priori power calculations were performed as we report a post hoc analysis using data from PROTECT-ASUC. Analyses were done using R V.4.0.2 and the survival package. ${ }^{8}$
This study was registered with research governance teams at all hospital sites to approve access to patient records. As no additional study procedures were carried out the need for written informed consent was waived by the ethics committee.

\section{RESULTS}

Of 822 patients recruited, we excluded 58 patients (1 coexisting COVID-19 infection, 6 fell outside study date periods, 19 did not receive any intravenous corticosteroid or rescue therapy, 26 with infective diarrhoea and 6 had missing data for primary outcome measure-colectomy) and report the outcomes in 764 patients divided into three groups: patients managed as inpatients for the entirety of their ASUC episode $(n=695)$, and an ambulatory cohort of seventy patients who are subdivided into patients managed as ambulatory from diagnosis 


\begin{tabular}{|c|c|c|c|c|c|}
\hline \multirow[b]{2}{*}{ Variable } & \multirow[b]{2}{*}{$\mathbf{n}$} & \multirow[b]{2}{*}{$\begin{array}{l}\text { Inpatient } \\
\mathrm{n}=695\end{array}$} & \multicolumn{2}{|l|}{ Ambulatory, $\mathrm{n}=69$} & \multirow[b]{2}{*}{$P$ value } \\
\hline & & & $\begin{array}{l}\text { Ambulatory from } \\
\text { diagnosis, } n=15\end{array}$ & $\begin{array}{l}\text { Initially inpatient and } \\
\text { then discharged to } \\
\text { ambulatory, } n=54\end{array}$ & \\
\hline Stool culture sent & 747 & $92.9 \%(631 / 679)$ & $64.3 \%(9 / 14)$ & $79.6 \%(43 / 54)$ & 0.0005 \\
\hline \multicolumn{6}{|c|}{ Stool frequency assessment on day 1 and day 3 after ASUC diagnosis } \\
\hline Stool frequency on both days & 583 & $75.2 \%(397 / 528)$ & $91.7 \%(11 / 12)$ & $74.4 \%(32 / 43)$ & \multirow[t]{2}{*}{0.5} \\
\hline Stool frequency on neither day & & $24.8 \%(131 / 528)$ & $8.3 \%(1 / 12)$ & $25.6 \%(11 / 43)$ & \\
\hline \multicolumn{6}{|c|}{ CRP tested on day 1 and day 3 after ASUC diagnosis } \\
\hline CRP on both days & 764 & 79.3\% (551/695) & $86.7 \%(13 / 15)$ & $72.2 \%(39 / 54)$ & \multirow[t]{3}{*}{0.62} \\
\hline CRP on one of days & & $14.0 \%(97 / 695)$ & $13.3 \%(2 / 15)$ & $20.4 \%(11 / 54)$ & \\
\hline CRP on neither days & & $6.8 \%(47 / 695)$ & $0.0 \%(0 / 15)$ & $7.4 \%(4 / 54)$ & \\
\hline $\begin{array}{l}\text { Underwent emergency flexible } \\
\text { sigmoidoscopy assessment }\end{array}$ & 756 & $77.3 \%(532 / 688)$ & $46.7 \%(7 / 15)$ & $84.9 \%(45 / 53)$ & 0.01 \\
\hline $\begin{array}{l}\text { Time to flexible sigmoidoscopy } \\
\text { (days) }\end{array}$ & 569 & $2.0(1.0-4.0)$ & $1.0(1.0-3.0)$ & $2.0(1.0-3.0)$ & 0.85 \\
\hline Discussed at IBD MDT & 746 & $38.7 \%(263 / 679)$ & $20.0 \%(3 / 15)$ & $32.7 \%(17 / 52)$ & 0.24 \\
\hline
\end{tabular}

$\mathrm{P}$ value=Fisher's exact test or Kruskal-Wallis test for discrete and continuous variables, respectively. Discrete variables displayed \% $(\mathrm{n} / \mathrm{N})$ and continuous variables median (IQR).

ASUC, acute severe ulcerative colitis; CRP, C reactive protein; IBD, inflammatory bowel disease; MDT, multidisciplinary team.

$(\mathrm{n}=15)$, and patients managed initially as inpatients but subsequently discharged to ambulatory pathways $(n=54)$ (figure 2). Of the 15 patients managed as ambulatory pathway from the point of diagnosis, $40 \%(6 / 15)$ were subsequently admitted to hospital; thus, $60 \%$ (9/15) patients received entirely ambulatory ASUC treatment. Inpatient ASUC cases were recruited from all 60 participating UK sites, whereas ambulatory patients were recruited from 22 centres (7 centres in prepandemic only, 10 centres in pandemic only and 5 centres in both eras) (online supplemental table 1).

\section{Baseline characteristics}

Inpatients, patients who were ambulatory from diagnosis and patients discharged to ambulatory pathways were well matched for gender, comorbidities, body mass index, IBD phenotype, years since IBD diagnosis and day 1 biomarkers (table 1). Ambulatory patients from diagnosis were younger (median age 30 years old (IQR 22-39) vs 37 years old (IQR 26-53) vs 45 years old (IQR 33-59), respectively, $\mathrm{p}=0.02$ ) than those managed as inpatients or discharged to ambulatory pathways.

\section{Initial assessment}

Patients who received ambulatory care from ASUC diagnosis were less likely to have a stool culture sent $(64 \%$ $(9 / 14)$ vs $80 \%(43 / 54)$ vs $93 \%(631 / 679)$, respectively, $\mathrm{p}=0.0005)$ or undergo a flexible sigmoidoscopy $(47 \%$ $(7 / 15)$ vs $85 \%(45 / 53)$ vs $77 \%$ (532/68), respectively, $\mathrm{p}=0.011)$ compared with patients who were discharged to ambulatory pathways or those patients receiving traditional inpatient care (table 2). Regarding other standards of ASUC assessment, the proportion of patients who underwent close monitoring of stool frequency and blood tests, timing of emergency flexible sigmoidoscopy and case discussion at IBD multidisciplinary meeting (MDT) were similar among the three groups.

\section{Outcomes}

The proportion of patients requiring colectomy during index ASUC management period was no different among all three treatment pathways: inpatient $=15.0 \%(104 / 695)$ vs ambulatory from diagnosis $=13.3 \%(2 / 15)$ vs discharged to ambulatory $=13.0 \%(7 / 54), \mathrm{p}=0.87$ (table 3 ). Additionally, response to intravenous corticosteroids, time to and rate of rescue or primary induction treatment (figure 3A), time to colectomy (figure 3B), mortality and duration of inpatient treatment were similar in the three groups (table 3). Patients treated as ambulatory from ASUC diagnosis were more likely to receive intravenous methylprednisolone rather than intravenous hydrocortisone than either patients discharged to ambulatory pathways or the inpatient cohort $(\mathrm{p}=0.0005)$. Eighteen $(2.6 \%$ $(18 / 690))$ of the inpatients but none of ambulatory cohort went straight to biologic rescue/induction without first receiving intravenous corticosteroids. Although not significant, there was a trend towards patients treated as ambulatory from diagnosis being more likely to receive rescue or primary induction treatment and yet they were less likely to respond to these therapies as compared with other treatment pathways.

\section{Three-month outcomes}

After 3-month follow-up from the index ASUC diagnosis, there was no significant difference in either rate of UC 
Ambulatory, $\mathrm{n}=69$

\begin{tabular}{|c|c|c|c|c|c|}
\hline Variable & $\mathbf{N}$ & $\begin{array}{l}\text { Inpatient } \\
\mathrm{n}=695\end{array}$ & $\begin{array}{l}\text { Ambulatory from } \\
\text { diagnosis, } n=15\end{array}$ & $\begin{array}{l}\text { Initially inpatient and } \\
\text { then discharged to } \\
\text { ambulatory, } n=54\end{array}$ & P value \\
\hline $\begin{array}{l}\text { Colectomy during index ASUC } \\
\text { management period }\end{array}$ & 764 & $15.0 \%(104 / 695)$ & $13.3 \%(2 / 15)$ & $13.0 \%(7 / 54)$ & 0.95 \\
\hline \multicolumn{6}{|l|}{ Secondary outcomes } \\
\hline \multicolumn{6}{|l|}{ Corticosteroid therapy } \\
\hline \multicolumn{6}{|l|}{ Received intravenous corticosteroids } \\
\hline Yes & 758 & $97.4 \%(672 / 690)$ & $100.0 \%(15 / 15)$ & $100.0 \%(53 / 53)$ & 0.74 \\
\hline No (straight to biologic) & & $2.6 \%(18 / 690)$ & $0.0 \%(0 / 15)$ & $0.0 \%(0 / 53)$ & \\
\hline Responded to corticosteroids & 753 & $71.6 \%(490 / 684)$ & $66.7 \%(10 / 15)$ & $75.9 \%(41 / 54)$ & 0.74 \\
\hline \multicolumn{6}{|l|}{ Rescue/primary induction therapy } \\
\hline $\begin{array}{l}\text { Received rescue/primary induction } \\
\text { therapy }\end{array}$ & 751 & $40.9 \%(281 / 687)$ & $60.0 \%(9 / 15)$ & $36.7 \%(18 / 49)$ & 0.28 \\
\hline $\begin{array}{l}\text { Time to rescue/primary induction } \\
\text { therapy (days) }\end{array}$ & 286 & $6.0(4.0-8.0)$ & $3.5(2.0-5.2)$ & $5.0(4.5-6.0)$ & 0.19 \\
\hline $\begin{array}{l}\text { Responded to rescue/primary } \\
\text { induction treatment }\end{array}$ & 301 & $80.4 \%(221 / 275)$ & $62.5 \%(5 / 8)$ & $88.9 \%(16 / 18)$ & 0.26 \\
\hline \multicolumn{6}{|l|}{ Surgery } \\
\hline
\end{tabular}

$P$ value=Fisher's exact test or Kruskal-Wallis test for discrete and continuous variables continuous, respectively. Discrete variables displayed $\%(\mathrm{n} / \mathrm{N})$ and continuous variables median (IQR).

*Only patients with ambulatory care from diagnosis who were admitted to hospital $(n=6)$ had inpatient stay data captured.

.ASUC, acute severe ulcerative colitis.

flare, readmission to hospital with UC flare or colectomy between the cohorts (table 4 ).

\section{DISCUSSION}

Approximately 1 in 10 patients diagnosed with ASUC are managed using an ambulatory pathway: for the majority this entails initial inpatient and then subsequent outpatient intravenous medical therapies; for a smaller number of patients treatment was successfully delivered entirely in the ambulatory setting. Although underpowered for our primary outcome, we report no difference in the requirement for colectomy among ASUC patients treated along ambulatory as compared with traditional inpatient pathways. Furthermore, 3 months after the index ASUC presentation, rehospitalisation for further flare and colectomy rates were not different. Although patients receiving ambulatory care from presentation were younger, we found no other differences in either baseline disease characteristics or biomarkers of ASUC severity.

Twenty-two out of sixty participating UK hospitals used an ambulatory pathway for one or more patients in the last 2 years. While COVID-19 related constraints on IBD services and concerns regarding nosocomial infection in immunosuppressed patients may have catalysed the use of ambulatory pathways, notably, 12 UK centres had already adopted this practice prior to outbreak of the pandemic.

The 2020 British Society of Gastroenterology (BSG) expert-based RAND panel guidelines for the management of ASUC during the COVID-19 pandemic specifically state that outpatient management with daily intravenous methylprednisolone is inappropriate, regardless of SARSCoV-2 status. ${ }^{9}$ Clinicians may be concerned that ambulant pathways hinder close observation, reduce the effectiveness of therapies and risk delays to treatment escalation. 
A

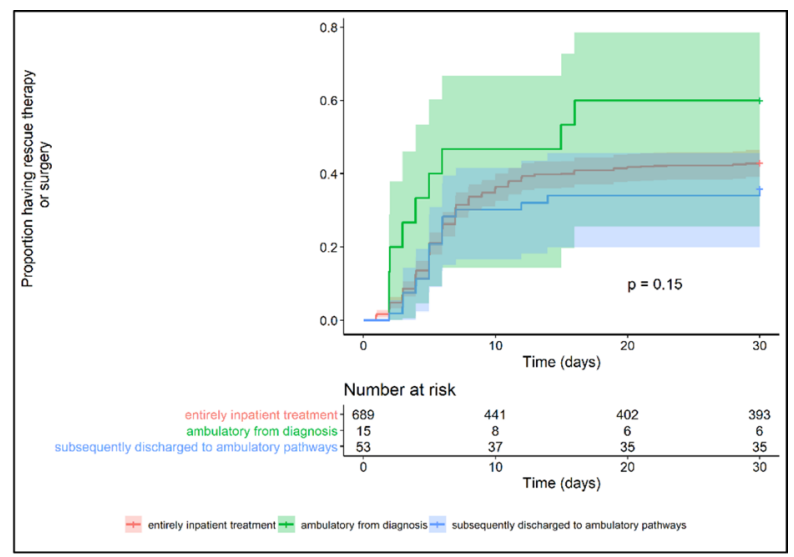

B

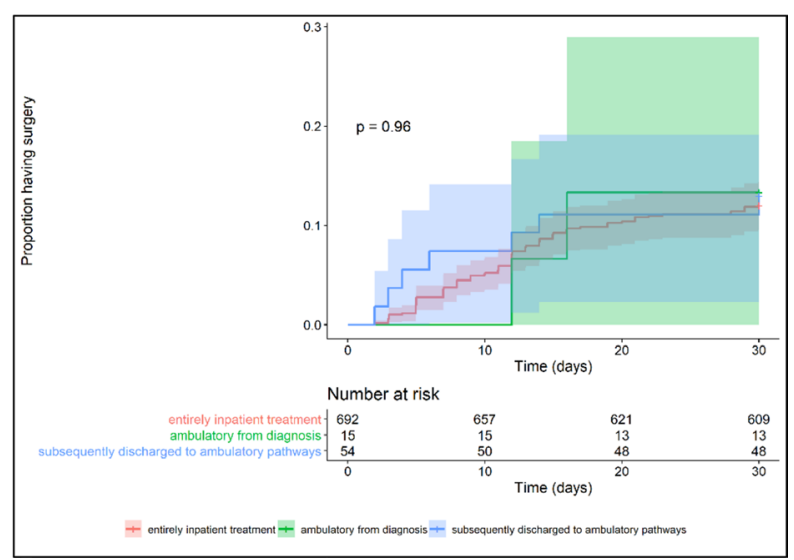

Figure 3 Time to initiation of rescue therapy or surgery for acute severe ulcerative colitis within the first 30 days $(A)$ and time to surgery (B).

We are concerned that stool culture and urgent flexible sigmoidoscopy were less frequently performed in the cohort treated as ambulatory from the point of diagnosis-these remain key investigations for the treatment of ASUC. ${ }^{10}$ However, other key standards including stool frequency clinical assessment, blood monitoring and IBD MDT discussion remained comparable in ambulatory and inpatient pathways, and there was no delay in escalation of treatment or time to surgery. Both methylprednisolone at doses of 1-1.5 mg/ $\mathrm{kg}$ (maximum $60 \mathrm{mg}$ / day) and hydrocortisone $100 \mathrm{mg}$ four times daily can be used in adult patients with ASUC with equal response rates. ${ }^{10}{ }^{11}$ In our study, physicians utilised methylprednisolone more in the ambulatory setting compared with the inpatient setting which likely reflects that methylprednisolone is a once-a-day medication compared with four times a day with hydrocortisone. Our rates for steroid response, rescue therapy and colectomy are all in line with published literature. ${ }^{12} 13$

Although our current data cannot be used to define a suitable cohort for ambulatory ASUC management, we speculate that patients with the following characteristics may be appropriate for such a pathway: younger patient age, motivated and engaged patient, no features of megacolon/imminent need for colectomy and biological naïve.

To the best of our knowledge, there are no studies reviewing management of patients with ASUC in the ambulatory setting prior to the pandemic, with just one single centre report detailing the benefits of such an approach for six patients as a COVID-19 pandemicdriven initiative. ${ }^{14}$ In this report, Townsend $e t$ al describe how they undertook daily patient review, clinical investigations and delivered intravenous corticosteroids, venous thromboembolism prophylaxis, and where appropriate, second-line infliximab rescue treatment all in an ambulatory setting. Only one of the six patients (all of whom met ASUC criteria) treated with this approach required hospital admission for colectomy. In our study, two ambulatory pathways were evident: for $80 \%$ of ambulatory patients this entailed initial inpatient intravenous corticosteroids, an expedited discharge and then further doses of intravenous corticosteroid as an outpatient; for $20 \%$ of ambulatory patients they received their first dose of intravenous corticosteroid as an outpatient. Notably, nearly half $(40 \%)$ of these latter ambulatory patients were subsequently admitted to hospital, demonstrating the importance of clear pathways for safe and urgent admission. Neither ambulatory approach was associated with detrimental outcomes.

There are several limitations to our dataset. First, in this post hoc analysis of data originally designed to capture adaptations to UK IBD care during the COVID-19

Table 4 Outcomes at 3-month follow-up period

\begin{tabular}{|c|c|c|c|c|c|}
\hline \multirow[b]{2}{*}{ Variable } & \multirow[b]{2}{*}{$\mathbf{N}$} & \multirow[b]{2}{*}{$\begin{array}{l}\text { Inpatient } \\
\mathrm{n}=695\end{array}$} & \multicolumn{2}{|l|}{ Ambulatory, $\mathrm{n}=69$} & \multirow[b]{2}{*}{$P$ value } \\
\hline & & & $\begin{array}{l}\text { Ambulatory from } \\
\text { diagnosis, } n=15\end{array}$ & $\begin{array}{l}\text { Initially inpatient and then } \\
\text { discharged to ambulatory, } \\
\mathrm{n}=54\end{array}$ & \\
\hline Experienced further UC flare & 678 & $27.6 \%(170 / 615)$ & $26.7 \%(4 / 15)$ & $20.8 \%(10 / 48)$ & 0.45 \\
\hline $\begin{array}{l}\text { Readmitted to hospital with further } \\
\text { UC flare }\end{array}$ & 632 & $25.0 \%(144 / 576)$ & $23.1 \%(3 / 13)$ & $27.9 \%(12 / 43)$ & 0.93 \\
\hline $\begin{array}{l}\text { Underwent colectomy (not including } \\
\text { index colectomy) }\end{array}$ & 686 & $4.5 \%(28 / 622)$ & $13.3 \%(2 / 15)$ & $4.1 \%(2 / 49)$ & 0.24 \\
\hline
\end{tabular}

$P$ value=Fisher's exact test or Kruskal-Wallis test for discrete and continuous variables continuous, respectively. Discrete variables displayed \% ( $\mathrm{n} / \mathrm{N})$ and continuous variables median (IQR).

UC, ulcerative colitis. 
pandemic, we acknowledge a relatively small number of patients in the ambulatory arms, meaning that our study is underpowered-especially after separation of ambulatory care into two subgroups. However, we thought the division of ambulatory groups justified as the two pathways are quite distinct and deserve separate comparison as such. Second, there is almost certainly a selection bias which biases towards the null in terms of our measured outcomes; this is evidenced by the highly selective use of ambulatory care pathways in most centres (where only one to two patients during the recruitment period were treated using an ambulatory pathway) and the younger age of patients selected by their physicians for ambulatory care treatment from the offset-although interestingly we found no other phenotypic differences between the treatment groups. Third, the ambulatory pathways used in each centre likely differ, although all ASUC patients must have received at least one dose of ntravenous corticosteroids in the outpatient setting to meet ambulatory criteria. Finally, we did not collect any patient feedback on the acceptability and perceived effectiveness of the ambulatory pathways.

\section{CONCLUSIONS AND RECOMMENDATIONS FOR FURTHER STUDIES}

This is the largest study of ambulatory ASUC treatment to date. We capture an emerging UK practice which challenges conventional treatment paradigms and current BSG expert guidance. We recommend that patients managed in the ambulatory setting are reviewed by gastroenterologists daily to monitor clinical parameters and assess for potential complications including venous thromboembolism and biochemical disturbance. We urge collaboration among UK centres and suggest establishing a prospective pragmatic non-inferiority study comparing colectomy rates in patients randomised to either ambulatory or inpatient treatment from the point of ASUC diagnosis. This study will explore both the clinical and cost effectiveness of ambulatory care as well as the patient and physician acceptability. These data may enable risk assessment and identification of patients based on baseline parameters suitable for ambulatory ASUC care; either entirely in an ambulatory setting, or where discharge can be expedited, with safe transfer of management to an ambulatory unit. Ambulatory ASUC care represents an ambitious target for transformational care within the IBD management.

\section{Author affiliations}

${ }^{1}$ IBD Unit, Department of Gastroenterology, Hull University Teaching Hospitals NHS Trust, Hull, UK

${ }^{2}$ Hull York Medical School, Hull, UK

${ }^{3}$ Department of Gastroenterology, St George's University Hospitals NHS Trust, London, UK

${ }^{4}$ Department of Gastroenterology, St Marks Hospital, London, UK

${ }^{5}$ Department of Gastroenterology, Royal Liverpool and Broadgreen University Hospitals NHS Trust, Liverpool, UK

${ }^{6}$ Univeristy of Liverpool, Liverpool, UK
${ }^{7}$ Gastroenterology, Royal Liverpool and Broadgreen University Hospitals NHS Trust, Liverpool, UK

${ }^{8}$ Gastroenterology, King's College Hospital NHS Foundation Trust, London, UK ${ }^{9}$ Gastroenterology, Kings College Hospital, London, UK

${ }^{10}$ Gastroenterology, Imperial College Healthcare NHS Trust, London, UK

${ }^{11}$ Gastroenterology, University College London Hospitals NHS Foundation Trust, London, UK

${ }^{12}$ Gastroenterology, University Hospital Birmingham NHS Foundation Trust, Birmingham, UK

${ }^{13}$ Institute of Applied Health Research, University Of Birmingham, Birmingham, UK

${ }^{14}$ Gastroenterology, Royal Wolverhampton NHS Trust, Wolverhampton, UK

${ }^{15}$ University of Wolverhampton, Wolverhampton, UK

${ }^{16}$ Newcastle University, Newcastle upon Tyne, UK

${ }^{17}$ Gastroenterology, Newcastle Upon Tyne Hospitals NHS Foundation Trust, Newcastle Upon Tyne, UK

${ }^{18}$ Gastroenterology, Royal Devon and Exeter NHS Foundation Trust, Exeter, UK

${ }^{19}$ IBD Pharmacogenetics, University of Exeter, Exeter, UK

${ }^{20}$ Gastroenterology, Torbay and South Devon NHS Foundation Trust, Torquay, UK

Twitter Shaji Sebastian @ibdseb and Jonathan P Segal @jonathansegal85

Acknowledgements We are grateful to the clinical and research teams in the participating sites for identification of patients, data collection and data entry. UK gastroenterology trainees and trainee networks: MaGNET-Mersey Gastroenterology Network, GLINT-Gastro London Investigative Network for Trainees, WMRIG - West Midlands Research in Gastroenterology, GasTRIN NoW-Gastroenterology Trainee Research and Improvement Network North-West, OxYGEN — The Oxford and Thames Valley Young Gastroenterologists Network TReNDD NI-Trainee Research Network in Digestive Diseases Northern Ireland) were integral in data collection for this study. We appreciate support from Crohn's \& Colitis UK and the British Society of Gastroenterology for promotion of this study.

Collaborators PROTECT ASUC collaboratorsThe entire group of collaborating authors has been submitted as a online supplemental file 2 .

Contributors SSe formed the study steering group. SSu and NAK was responsible for initial study design which was further developed by the steering group. NAK and GW led methodological development and all members of the steering group contributed to subsequent protocol development. SSe led regulatory approvals and study co-ordination. The PROTECT-ASUC study group were responsible for local site approvals, data acquisition and data entry. GW and NK led the statistical analysis supported by all members of the steering group. KVP, JS, SSu and GW led the writing group. All members of the steering group contributed to manuscript redrafting, editing and review and approved the final version. SSe is the guarantor of the study

Funding The authors have not declared a specific grant for this research from any funding agency in the public, commercial or not-for-profit sectors.

Competing interests SS holds research grants from Biogen, Takeda, AbbVie, Tillotts Pharma, Ferring and Biohit; served on the advisory boards of Takeda, AbbVie, Merck, Ferring, Pharmacocosmos, Warner Chilcott, Janssen, Falk Pharma, Biohit, TriGenix, Celgene and Tillots Pharma; and has received speaker fees from AbbVie, Biogen, AbbVie, Janssen, Merck, Warner Chilcott and Falk Pharma. GW has served as a speaker and/or advisory board member for AbbVie, Falk and Janssen. He has had support to attend meetings from AbbVie, Falk, Janssen and Norgine. His department has received research funding from Tillotts. NAK has served as a speaker and/or advisory board member for Allergan, Falk, Janssen, Mylan, Pharmacosmos, Takeda and Tillotts. He has had support to attend meetings from AbbVie, Falk, Janssen and Norgine. His department has received research funding from AbbVie, Celgene, Celtrion, MSD, Napp, Pfizer, Pharmacosmos and Takeda. SSu has received speaker fees from MSD, Actavis, Abbvie, Dr Falk pharmaceuticals, Shire and received educational grants from MSD, Abbvie, Actavis and is an advisory board member for Celltrion, Dr Falk pharmaceuticals and Vifor pharmaceuticals. CAL has received research support and/or has received fees for delivery of non-promotional education from: Genentech, Janssen, Takeda, Abbvie, Dr Falk, AstraZeneca, Eli Lilly, Orion, Pfizer, Roche, Sanofi Aventis, Ferring, UCB and Biogen. MJBB has received research grants from Vifor International, Pharmacosmos and Tillots Pharma; has received speaker fee from Abbvie and Vifor International; has been an advisory board member for Tillots Pharma, Vifor International; and received travel/conference expenses from Vifor International, Abbvie and Tillots Pharma. AK has served on the advisory boards for Abbvie, Janssen and BMS Celgene and has received speaker fees from Takeda, Pfizer and Janssen; and received travel/ conference expenses from Tillotts, Janssen, Abbvie and Shield Therapeutics. KVP 
has received honoraria for educational meetings and speaker fees from Abbvie, Janssen, Takeda, DrFalk and Ferring. KVP has received Advisory Board fees from Abbvie and Janssen.

Patient consent for publication Not applicable.

Ethics approval The study was approved by Leeds and Bradford ethics committee (IRAS No:284030, REC reference:20/HRA/2578).

Provenance and peer review Not commissioned; externally peer reviewed.

Data availability statement All data relevant to the study are included in the article or uploaded as online supplemental information.

Supplemental material This content has been supplied by the author(s). It has not been vetted by BMJ Publishing Group Limited (BMJ) and may not have been peer-reviewed. Any opinions or recommendations discussed are solely those of the author(s) and are not endorsed by BMJ. BMJ disclaims all liability and responsibility arising from any reliance placed on the content. Where the content includes any translated material, BMJ does not warrant the accuracy and reliability of the translations (including but not limited to local regulations, clinical guidelines, terminology, drug names and drug dosages), and is not responsible for any error and/or omissions arising from translation and adaptation or otherwise.

Open access This is an open access article distributed in accordance with the Creative Commons Attribution Non Commercial (CC BY-NC 4.0) license, which permits others to distribute, remix, adapt, build upon this work non-commercially, and license their derivative works on different terms, provided the original work is properly cited, appropriate credit is given, any changes made indicated, and the use is non-commercial. See: http://creativecommons.org/licenses/by-nc/4.0/.

\section{ORCID iDs}

Shaji Sebastian https://orcid.org/0000-0002-3670-6545

Kamal V Patel https://orcid.org/0000-0002-9668-0316

Jonathan P Segal https://orcid.org/0000-0002-9668-0316

Sreedhar Subramanian https://orcid.org/0000-0002-64831730

Thomas Edward Conley https://orcid.org/0000-0002-0604-0770

Haidee Aleman Gonzalez https://orcid.org/0000-0003-0559-0807

Alexandra J Kent https://orcid.org/0000-0003-0577-6177

Aamir Saifuddin https://orcid.org/0000-0002-5888-5556

Lucy Hicks https://orcid.org/0000-0002-3065-1635

Shameer Mehta https://orcid.org/0000-0002-7002-293X

Neeraj Bhala https://orcid.org/0000-0003-2502-1177

Matthew J Brookes https://orcid org/0000-0002-8782-0292

Christopher A Lamb https://orcid.org/0000-0002-7271-4956

Nicholas A Kennedy http://orcid.org/0000-0003-4368-1961

Gareth J Walker https://orcid.org/0000-0002-3883-8816

\section{REFERENCES}

1 Domènech $E$, Mañosa $M$, Cabré $E$. An overview of the natural history of inflammatory bowel diseases. Dig Dis 2014;32:320-7.

2 Dong C, Metzger M, Holsbø E, et al. Systematic review with meta-analysis: mortality in acute severe ulcerative colitis. Aliment Pharmacol Ther 2020;51:8-33.

3 Seah D, De Cruz P. Review article: the practical management of acute severe ulcerative colitis. Aliment Pharmacol Ther 2016;43:482-513.

4 Kennedy NA, Hansen R, Younge L, et al. Organisational changes and challenges for inflammatory bowel disease services in the UK during the COVID-19 pandemic. Frontline Gastroenterol 2020;11:343-50.

5 Aujesky D, Roy P-M, Verschuren F, et al. Outpatient versus inpatient treatment for patients with acute pulmonary embolism: an international, open-label, randomised, non-inferiority trial. Lancet 2011;378:41-8

6 Zondag W, Kooiman J, Klok FA, et al. Outpatient versus inpatient treatment in patients with pulmonary embolism: a meta-analysis. Eur Respir J 2013;42:134-44.

7 Sebastian S, Walker GJ, Kennedy NA, et al. Assessment, endoscopy, and treatment in patients with acute severe ulcerative colitis during the COVID-19 pandemic (PROTECT-ASUC): a multicentre, observational, case-control study. Lancet Gastroenterol Hepatol 2021;6:271-81.

8 Therneau TM. Survival Analysis [R package survival version 3.2-7], 2020.

9 Din S, Kent A, Pollok RC, et al. Adaptations to the British Society of gastroenterology guidelines on the management of acute severe Uc in the context of the COVID-19 pandemic: a Rand appropriateness panel. Gut 2020;69:1769-77.

10 Lamb CA, Kennedy NA, Raine T, et al. British Society of gastroenterology consensus guidelines on the management of inflammatory bowel disease in adults. Gut2019;68:s1-106.

11 Lamb CA, Kennedy NA, Raine T, et al. British Society of gastroenterology consensus guidelines on the management of inflammatory bowel disease in adults. Gut 2019;68:s1-106.

12 Williams JG, Alam MF, Alrubaiy L, et al. Infliximab versus ciclosporin for steroid-resistant acute severe ulcerative colitis (construct): a mixed methods, open-label, pragmatic randomised trial. Lancet Gastroenterol Hepatol 2016;1:15-24.

13 Narula N, Marshall JK, Colombel J-F, et al. Systematic review and meta-analysis: infliximab or cyclosporine as rescue therapy in patients with severe ulcerative colitis refractory to steroids. Am J Gastroenterol 2016;111:477-91.

14 Townsend T, Fiske J, Collins P, et al. Ambulatory management of acute severe ulcerative colitis: a Pandemic-driven initiative. Inflamm Bowel Dis 2020;26:e112-3. 